\title{
DISCLAIMER
}

This report was prepared as an account of work sponsored by an agency of the United States Government. Neither the United States Government nor any agency thereof, nor any of their employees, makes any warranty, express or implied, or assumes any legal liability or responsibility for the accuracy, completeness, or usefulness of any information, apparatus, product, or process disclosed, or represents that its use would not infringe privately owned rights. Reference herein to any specific commercial product, process, or service by trade name, trademark, manufacturer, or otherwise does not necessarily constitute or imply its endorsement, recommendation, or favoring by the United States Government or any agency thereof. The views and opinions of authors expressed herein do not necessarily state or reflect those of the United States Government or any agency thereof.

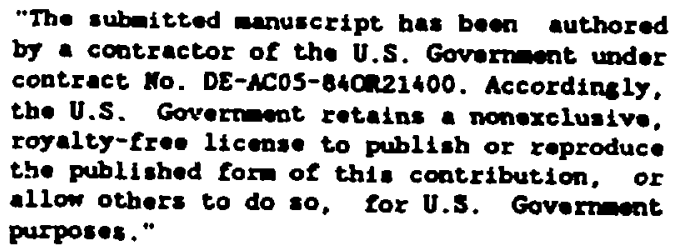




\section{Design of high-reflectivity supermirror structures}

John B. Hayter and H. A. Mook

Solid State Division, Oak Ridge National Laboratory

P.0. Box 2008, Oak Ridge, TN 37831-6031

\section{ABSTRACT}

We present, a new method of designing supermirrors, based on considering the contribution of each bilayer to the extinction in a given stack of bilayers, and derive and solve the discrete equations governing the choice of layer thicknesses. In the limit of zero layer thickness, the usual continuum result is recovered. The design, which can produce essentially perfect reflectivity over the entire supermirror range, also provides an objective physical mechanism for trading reflectivity to gain angular range.

\section{INTRODUCTION}

Many of the optical techniques developed for use with visible light can be employed directly with neutron or $X$-radiation ${ }^{1}$, requiring only an appropriate formalism for computing the refractive indices 2,3 . In the case of neutron optics, an extensive current application lies in the transport of neutrons over many tens of meters through guides, which make use of total external reflection on thin film coatings on the interior walls of an evacuated tube, usually of rectangular cross-section. A fundamental limitation of such guides is the small solid angle $\left(4 \theta_{\mathrm{C}}^{2}\right)$ which may be transported, due to the intrinsically snall total reflection angles, $\theta_{c}$, which are available from uniform thin films. To overcome this situation, Mezei ${ }^{4}$ proposed a modified multilayer thin-film design, termed a supermirror, to extend the angular range reflected by the mirror coating, using an aperiodic structure, in which successive bilayers vary in thickness, analogous to the designs used in light optics for broad-band filter design5. To date, designs for supermirrors $4,6-11$ have been based on continuum approaches. In this paper, we derive a discrete design technique which takes into specific account the discontinuous nature of the structure.

\section{PERIODIC BILAYER STRUCTURES}

As a necessary̆ prelininary step, prior to considering a general supermirror, we shall consider the case of periodic multilayer structures, first establishing the general optical relations common to any type of thin-film structure. We write the refractive index for a given material in the generic form

$$
n=1-(\alpha-i \beta)
$$

where, at wavelength $\lambda$,

$$
\alpha-\left(N \lambda^{2} / 2 \pi\right)\left(b_{0}+\Delta b^{\prime}\right) ; \quad \beta=\left(N \lambda^{2} / 2 \pi\right)\left|\Delta b^{\prime \prime}\right|
$$

for neutrons (scattering amplitude $b_{0}+\Delta b^{\prime}-i \Delta b^{n}$ ), and 


$$
\alpha=\left(N \lambda^{2} r_{e} / 2 \pi\right)\left(f_{0}+\Delta f^{\prime}\right) ; \quad \beta-\left(N \lambda^{2} r_{e} / 2 \pi\right)\left|\Delta f^{\prime \prime}\right|
$$

for X-rays. (scattering amplitude $\left.\left(f_{0}+\Delta f^{\prime}+i \Delta f^{\prime \prime}\right) r_{e}\right)$, where $r_{c}$ is the classical electron radius. Here, $N$ is the number density of scatterers in the material; scattering amplitudes are available in tabulated form 12,13 . For neutrons, we shall usually have $\beta \simeq 0$, since strongly absorbing materials will generally not be of interest for mirror design, although reflection from strongly absorbing layers must be considered in, for example, collimator design. The situation is different for $X$-rays, since absorption is not so easily avoided, and we may commonly expect $\beta / \alpha$ values up to about 0.1 in the $X$-ray case.
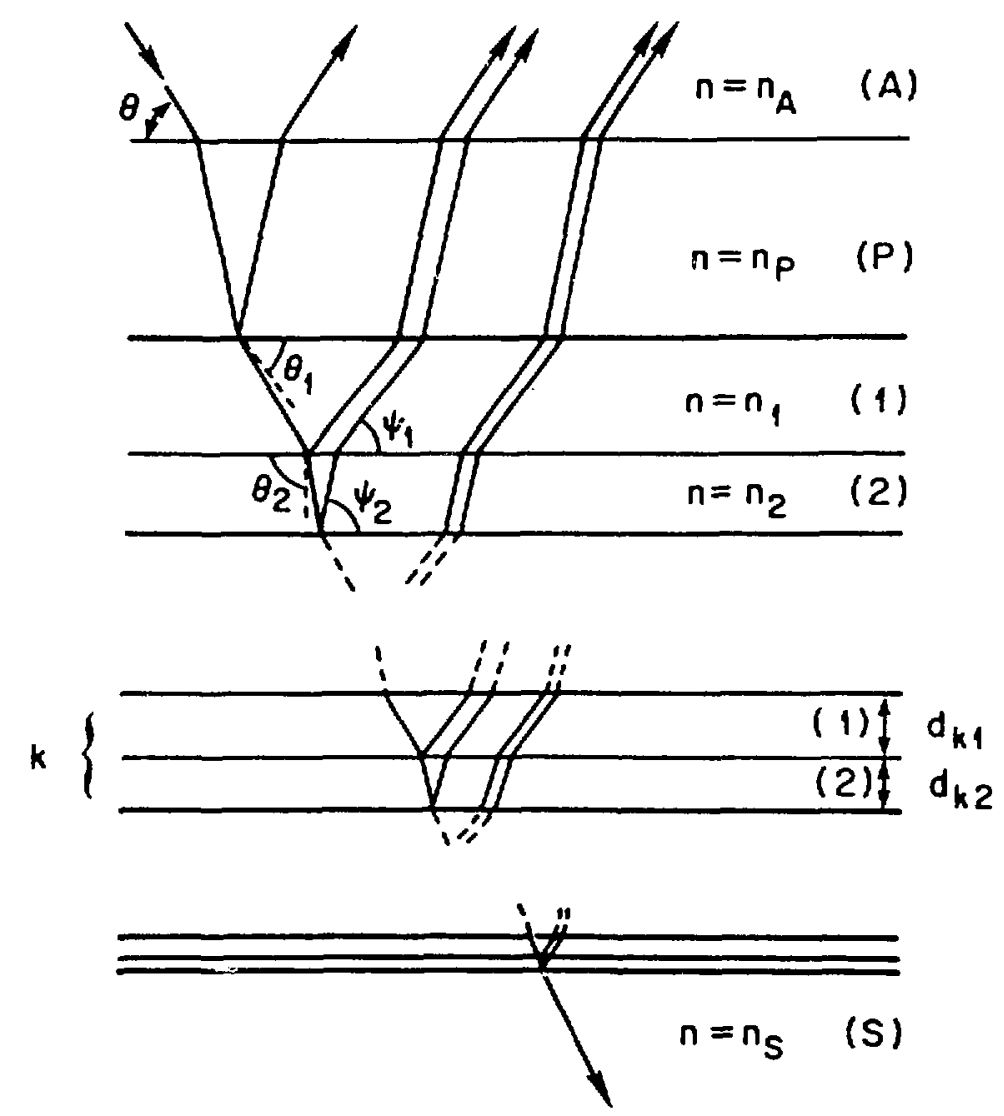

Figure 1. Definition of multilayer geometry. The structure comprises $2 \mathrm{~N}$ alternating films of materials labelled types $I$ and 2 , having respective refractive indices $n_{1}$ and $n_{2}$, and thicknesses $d_{k 1}$ and $d_{k 2}$ in the $k^{\prime}$ th bilayer $(k-1,2, \ldots, N)$.

Figure 1 defines our notation for a general discrete thin-film multilayer (DTFM) structure, in which successive bilayers of materials (generically labelled types 1 and 2) are deposited on a substrate, $S$; the structure may be protectad from the atmosphere $A$ by a layer $F$. All angles are sufficiently small to permit the approximations $\sin \theta \approx \theta, \cos \theta \approx 1-\theta^{2}$. Consider a plane wave, $\exp \left(i k_{0} \cdot r\right)$, incident on the multilayer structure at an angle, $\varphi$, which is larger than that for total reflection, so that the wave enters the structure. In a layer of material type $\mu(\mu=1,2)$, of refractive index $n_{\mu}$, we shall have an inhomogeneous plane wave, whose planes of constant phase are normal to the direction defined by the angle $\psi_{\mu}$. where ${ }^{3}$ 


$$
\psi_{\mu}^{2}=\left\{\left[\left(\theta^{2}-\gamma_{\mu}^{2}\right)^{2}+4 \beta_{\mu}^{2}\right]^{1 / 2}+\theta^{2}-\gamma_{\mu}^{2}\right\} / 2
$$

Here, we have defined $\gamma_{\mu}^{2}-2 \alpha_{\mu}$. In the absence of absorption, $\gamma_{\mu}$ will be the critical angle for total reflection at a vacuum- $\mu$ interface, and $\psi_{\mu}$ will coincide with the angle $\theta_{\mu}$ given by Snell's law; generally, however, the latter is complex, while $\psi_{\mu}$ is real. Optimal reflectivity will occur when the bilayer is a $\lambda / 2$ plate, with each component sub-layer being a $\lambda / 4$ plate. Since $|n-1| \approx 0, k_{\mu} \approx k_{0}$, and $\theta$ will be a Bragg angle when

$$
d_{\mu}-\lambda_{0} / 4 \psi_{\mu}
$$

Eqs. (3) and (4) give the refraction-corrected expression of Bragg's law for the bilayer. Without loss of generality, we may now restrict further discussion to the case $\gamma_{1} \gamma_{\gamma_{2}}$.

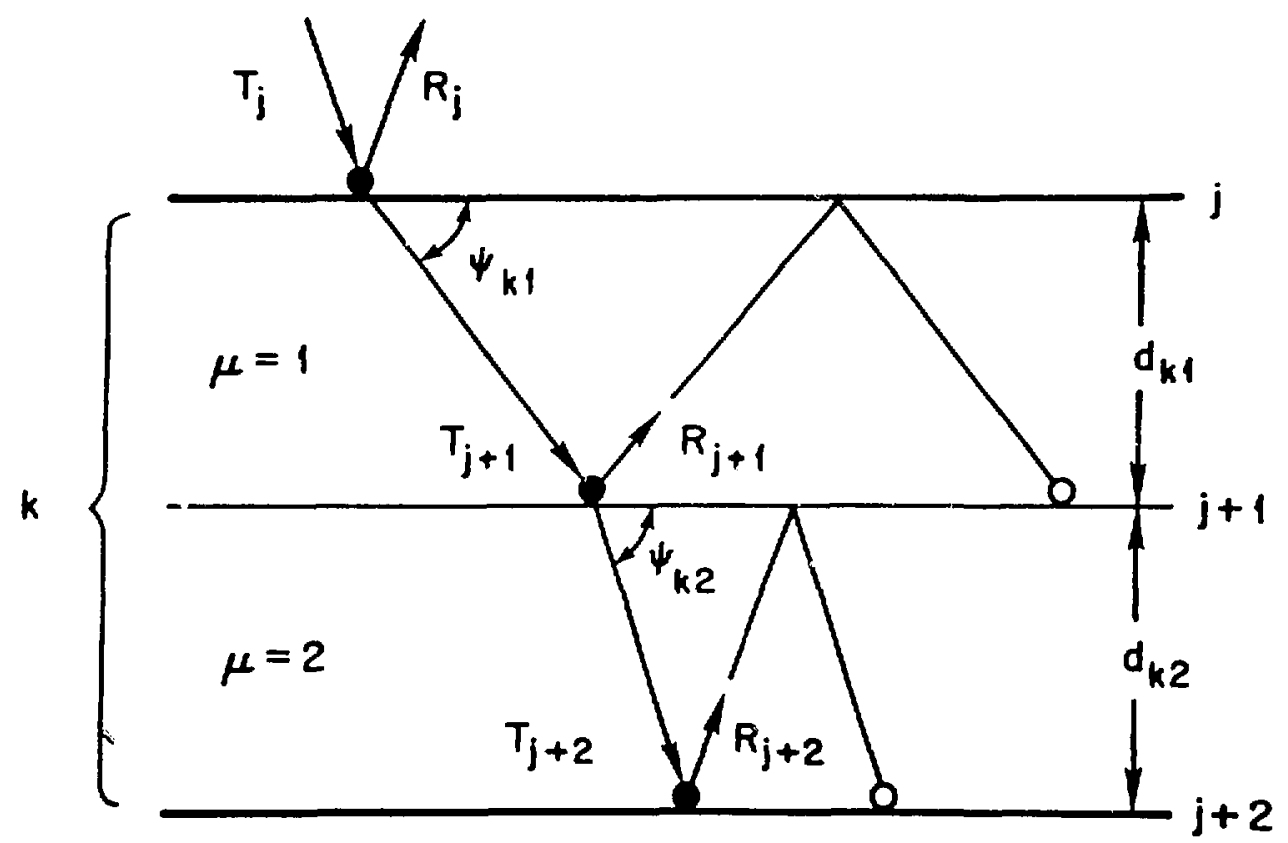

Figure 2. Multiple beams in the $k^{\prime}$ th bilayer of a multi-bilayer stack.

For a general ray in the structure, the multiple beam equations are established as follows. Let $T_{j}, R_{j}$ be the transmitted and reflected amplitudes just above the $j^{\prime}$ th interface (see Figure 2), where $j$ is even at 2-1 interfaces, and odd at 1-2 interfaces. Writing the reflection and transaission coefficients at the 2-1 interface as $r_{j}$ and $t_{j}$, respectively, we may construct the amplitude relations by noting that $T$ is composed of a directly transmitted amplitude plus a retransmitted, doubly reflected amplitude, with a similar inverse consideration for $R$. Taking into account the phase shift between 2-1 and 1-2 reflections, the amplitude relations are thus

$$
\begin{aligned}
& R_{j}=r_{j} T_{j}+t_{j} R_{j+1} \exp \left(i \phi_{j+1}\right) . \\
& T_{j+1}=t_{j} T_{j} \exp \left(i \phi_{j+1}\right)+\bar{r}_{j} R_{j+1} \exp \left(2 i \phi_{j+1}\right)
\end{aligned}
$$




$$
\begin{aligned}
& R_{j+1}=\bar{r}_{j+1} T_{j+1}+t_{j+1} R_{j+2} \exp \left(i \phi_{j+2}\right) \\
& T_{j+2}=t_{j+1} T_{j+1} \exp \left(i \phi_{j+2}\right)+r_{j+1} R_{j+2} \exp \left(2 i \phi_{j+2}\right) .
\end{aligned}
$$

where $\phi_{j}=k_{0} d_{j} \psi_{j}$ is the normal component of the phase difference between interface $j$ and interface $j+1$, and $\bar{r}$ is the reflection coefficient at the $1-2$ interface. The reflection and transmission coefficients are given by the usual fresnel relations 1 , noting that conservation of energy requires $r^{2}+t^{2}-1$ :

$$
\begin{aligned}
& r=-\bar{r}=\rho-\left(\psi_{2}-\psi_{1}\right) /\left(\psi_{2} \cdot \psi_{1}\right) \\
& \tau=2\left(\psi_{1} \psi_{2}\right)^{1 / 2}\left(\left(\psi_{2}+\psi_{1}\right)\right.
\end{aligned}
$$

The equations (5) are closed by imposing the periodic boundary conditions which result from the fact that the bilayer composition is independent of the bilayer number in the present case. This means that the relative attenuation per bilayer, $\kappa$, is constant, so that

$$
\begin{aligned}
\kappa & -T_{j+2} / T_{j} \\
& =R_{j+2} / R_{j}
\end{aligned}
$$

Equations (5-7) may be solved exactly using standard techniques. (At small angles, the reflectivity of typical bilayers is not small, and it is worth noting that the usual expansion techniques are inapplicable.) A lengthy calculation yields

$$
\kappa=-\left(1-\rho^{2}\right) \exp \left[i\left(\phi_{1}+\phi_{2}\right)\right] /\left[y-1+\rho^{2} \exp \left(2 i \phi_{1}\right)\right]
$$

where $y$ is the solution of

$$
\begin{aligned}
& y^{2}-\left[1-p^{2}\left[\exp \left(2 i \phi_{1}\right)-\exp \left(2 i \phi_{2}\right)\right]-\exp \left[2 i\left(\phi_{1}+\phi_{2}\right)\right]\right\} y \\
& +\rho^{2} \exp \left(2 i \phi_{2}\right)\left[1-\exp \left(2 i \phi_{1}\right)\right]^{2}=0
\end{aligned}
$$

which satisfies $\left|R_{\mathrm{o}}\right| \leq 1$. Defining $T_{\mathrm{o}}=1$ as the reference amplitude, the reflectivity of the entire stack is

$$
R_{0}=y /\left(\rho\left[1-\exp \left(2 i \phi_{1}\right)\right] \exp \left(2 i \phi_{2}\right)\right\}
$$

At the Bragg condition, $\phi_{1}=\phi_{2}-\pi / 2$, and for angles near the Bragg condition we may take $\phi_{1} \approx \phi_{2} \approx \phi$. Equation (9) may then be solved explicitly, yielding the reflectivity

$$
R_{0}=\left[1+\exp (2 i \phi)+2 \exp (i \phi)\left(\cos ^{2} \phi-\rho^{2}\right)^{1 / 2}\right] /[2 \rho \exp (2 i \phi)]
$$

from which we obtain the reflected intensity:

$$
\begin{array}{ll}
\left|R_{\mathrm{o}}\right|^{2}=1 & \left(\cos ^{2} \phi \leq \rho^{2}\right) \\
\left|R_{\mathrm{o}}\right|^{2}=2 \cos \phi\left[\cos \phi+\left(\cos ^{2} \phi-\rho^{2}\right)^{1 / 2}\right] / \rho^{2}-1 & \left(\cos ^{2} \phi>\rho^{2}\right)
\end{array}
$$

The half-intensity points are given by

$$
\cos \phi_{1 / 2}-3 \rho / 2 \sqrt{2}
$$


from which the well-known Darwin result is recovered when the phase-shifts in each half of the bilayer are the same.

\section{VARIABLE THICKNESS MULTI-BILAYER}

We now consider an arbitrary bilayer, $k$, in a supermirror stack (Figure 1), and choose the angle of incidence, $\theta_{k}$, of the beam arriving on the stack, such that it meets the Bragg condition defined by eqs. (3) and (4) for the layer in question. If the stack were uniform, the amplitude attenuation through the bilayer would be given by eqs. (8) and (9) with $\phi_{k 1}-\phi_{k 2}-\pi / 2$, namely (uropping subscripts for clarity)

$$
\kappa=-(l-\rho) /(1+\rho)=-\psi_{1} / \dot{\psi}_{2}
$$

where the - sign reflects the $\pi$ phase shift across a bilayer at the Bragg condition. Let $\nu$ bilayers contribute to the reflection; that is, after $\nu$ bilayers, the transmitted intensity, $\left|\kappa^{\nu}\right|^{2}$, is small enough to be essentially negligible. Consider now removing the bilayer from the uniform stack, and placing it in a stack of bilayers of different thicknesses, such that it is effectively the only layer reflecting at its own Bragg angle. Without the reinforcement of the periodic boundary conditions, it will now attenuate the transmitted intensity by as amount, $|\bar{\kappa}|^{2}$, which is essentially the $\nu^{\prime}$ th root of its previous performance. Assigning it an effective reflectivity of $\bar{\rho}$ in the aperiodic system, we thus have

$$
|\bar{\kappa}|=(1-\bar{\rho}) /(1+\bar{\rho})=|\kappa|^{1 / \nu}
$$

from which

$$
\bar{\rho}=\left(1-|\kappa|^{1 / \nu}\right) /\left(1+|\kappa|^{1 / \nu}\right)
$$

We may assess $\nu$ by quantifying what we mean by "negligible" transmitted intensity after $\nu$ bilayers. Let us require a fraction 5 of the incident intensity to be reflected, so that the transmitted intensity is

$$
1-5=\left|x^{\nu}\right|^{2}
$$

which yields (subject to the obvious physical restriction $\nu \geq 1$ )

$$
\nu=\ln (1-5) /(2 \ln [(1-\rho) /(1+\rho)])
$$

The width of the Bragg reflection from the single layer in the aperiodic stack is then essentially given by eq. (13), with $\rho$ replaced by $\bar{\rho}$ computed from eqs. (15), (16) and (18). Replacing the subscript $k$ to emphasize the dependence on bilayer number, eq. (13) becomes

$$
\cos \left[(\pi / 2)\left(\psi_{k \mu} \mp \Delta \psi_{k \mu}\right) / \psi_{k \mu}\right]-3 \bar{\rho}_{k} / 2 \sqrt{ } 2
$$

or, inverting the trigonometric function,

$$
\Delta \psi_{k \mu} / \psi_{k \mu}- \pm(2 / \pi) \arcsin \left(3 \bar{p}_{k} / 2 \sqrt{2}\right)
$$

where we have used eq. (4) to evaluate $\phi$. Here, $\phi_{k \mu}$ is the value of $\phi$ at the Bragg condition corresponding to the thickness of material $\mu$ in the $k^{\prime}$ th bilayer, and $\Delta \psi_{k \mu}$ is the offset at which the intensity is halved. 
To find the corresponding values of $\theta_{k}$, it is convenient to use scaled parameters

$\tau_{k}-\theta_{k} / \gamma_{1}$

$\omega_{k \mu}-\psi_{k \mu} / \gamma_{1}$

$g_{\mu}^{2}=\alpha_{\mu} / \alpha_{1}$

$h_{\mu}=\beta_{\mu} / \alpha_{1}$

in terms of which eq. (3) becomes

$w_{k \mu}^{2}=\left(\left[\left(\tau_{k}^{2}-g_{\mu}^{2}\right)^{2}+h_{\mu}^{2}\right]^{2 / 2}+\tau_{k}^{2}-g_{\mu}^{2}\right) / 2$

We now substitute $t \Delta r$ for $r$, and $t \Delta w$ for $w$, in eq. (22), and solve for the variation $\Delta r$ which corresponds to the variation $\Delta w$. This yields for the halfintensity points at the vacuum (or, in practice, air) interface

$$
\tau_{k \pm \Delta \tau_{k}}( \pm)-\frac{\left(4\left(w_{k \mu} \pm \Delta w_{k \mu}\right)^{2}\left[\left(w_{k \mu} \pm \Delta w_{k \mu}\right)^{2}+g_{\mu}^{2}\right]-h_{\mu}^{2}\right)^{1 / 2}}{2\left(w_{k \mu} \pm w_{k \mu}\right)}
$$

where

$$
\Delta w_{k \mu}=\left(2 w_{k \mu} / \pi\right) \arcsin \left(3 \bar{\rho}_{k} / 2 \sqrt{2}\right)
$$

and the superscripts ( \pm ) reflect the asymmetry of the intensity profile when expressed as a function of $\boldsymbol{r}_{k}$.

To construct a supermirror stack, the thicknesses of successive bilayers are now chosen such that their reflectivity profiles match at the half-intensity points:

$$
\tau_{k}-\Delta \tau_{k}^{(-)}=\tau_{k-1}+\Delta \tau_{k-1}(+)
$$

Eq. (25), which prescribes the geometry of bilayer $k$, in terms of that of bilayer $k-1$. is the central result of this paper. The equation is readily solved for $\boldsymbol{r}_{k}$ in terms of ${ }^{\top} k-I$ by simple Newton iteration, which is rapidly convergent; since the left hand side of eq. (23) does not depend on $\mu$, we need only solve for $\mu=1$, which then completely defines the bilayer geometry through eqs. (4), (21) and (22). The entire supermirror structure may thus be built up layer by layer, given a suitable initial condition to start the process. The obvious choice is to match the first superairror layer $(k-1)$ to the half-intensity point of the total mirror reflection:

$$
r_{0}+\Delta r_{0}(t)=3 / 2 \sqrt{ } 2
$$

The design is fully deternined by the refractive indices of the bilayer naterials, once the reflectivity parameter, 5 , has been specified. It is interesting to note that, at sufficiently high incident angles, the reflecting layers vary only slowly in thickness. In this limit, we may express eq. (25) as

$$
\partial r_{k} / \partial k \approx 2 \Delta r_{k}
$$

Expanding eq. (23) for the case $r_{k}>1$, substituting in (27) and integrating yields 


$$
d_{k \mu} \propto\left(\lambda_{0} / 4 \gamma_{\mu}\right) k^{-1 / 4}
$$

which shows that the continuum result 6,7 corresponds. to the case of vanishing layer thickness, as expected.

\section{RESULTS AND DISCUSSION}

The main features of the DTFM design appear in Figure 3 . First, total reflection is maintained close to the critical reflection edge, where the discrete nature of the structure is most important. The response of a Ni-Mn DTFM structure of only 8 bilayers is shown in Figure 3a, where it is seen that a gain of 358 in the range of total reflectivity is achieved, at a very modest cost in complexity, compared with a natural $\mathrm{Ni}$ mirror; for comparison, the use of the pure isotope ${ }^{58_{\mathrm{Ni}}}$ would provide a gain factor of only 188. Curves $b$ and $c$ of Figure 3, which both show gain factors of 3 over a $\mathrm{Ni}$ mirror, compare the continuum design (Figure $3 b$ of Schelten Mika $^{9}$ ) with the DTFM design (Figure 3c). The DTFM shows no losses near the critical edge, and is better optimized, achieving a better response in 350 layers than does the continuum design in 404 layers.

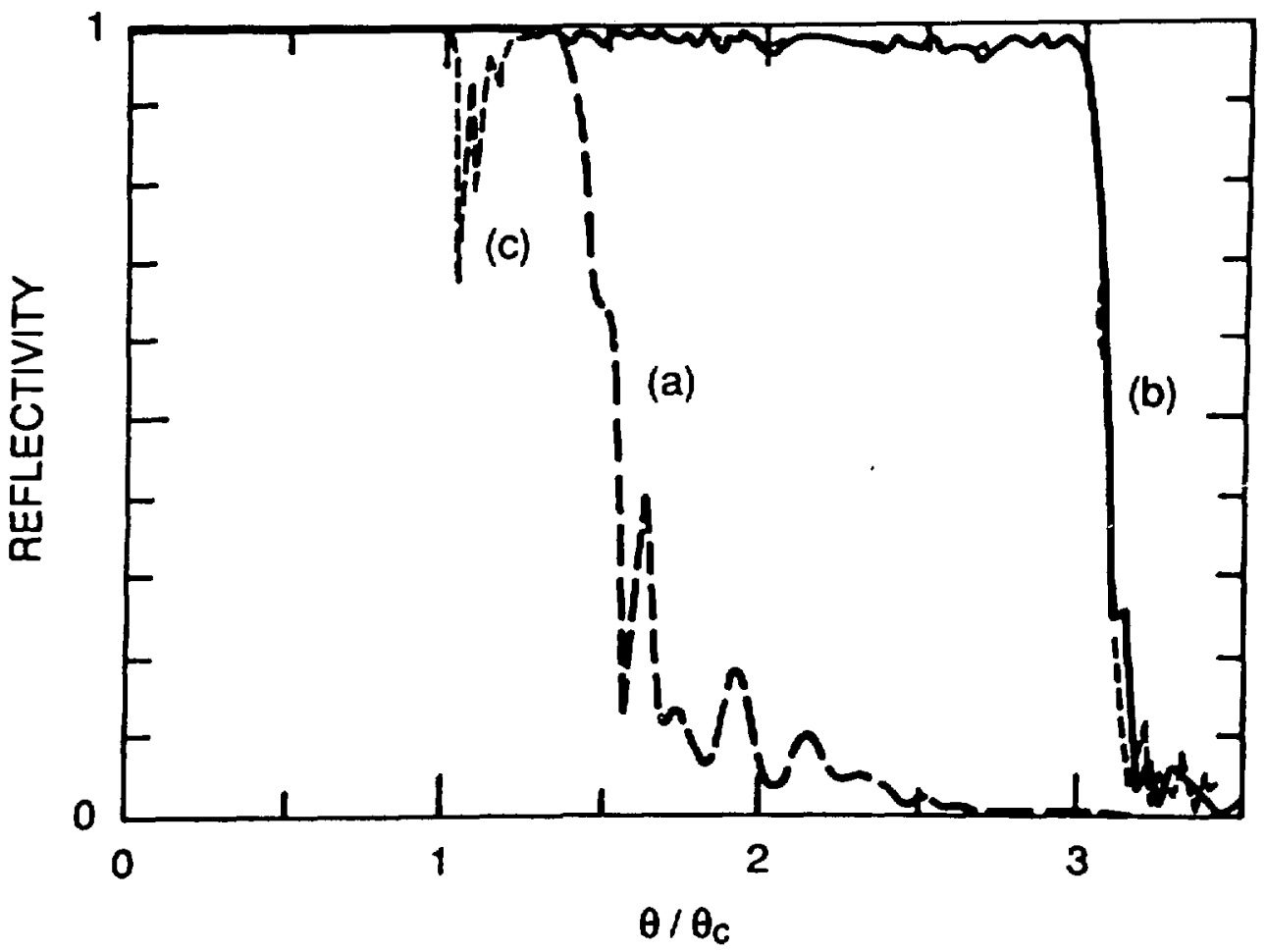

Figure 3. Calculated 14,15 neutron reflectivities of $\mathrm{Ni} / \mathrm{Mn}$ supermirrors, shown as a function of glancing angle, $\theta$, relative to the critical angle for total mirror reflection, $\theta_{c}$. (a) DTFM $(5-0.988)$ with 16 layers total; (b) 350 layer DTFM; (c) 404 layer continuun design of Schelten and Mika ${ }^{9}$.

The effective orthogonality of each layer generated by eq. (25) allows the construction of bandpass filters, since layers may be onitted from a full DTFM design without deteriorating the response elsewhere. Such designs may be of interest in the fabrication of long wavelength monochromators for use in biochemical 
crystallography. We note in this context that the analysis of $\$ 2$ is quite general, and applies to synthetic thin-film monochromator designs 16,17 , in which all bilayers have the same thicknesses. (The term multilayer has become generally reserved for such synthetic crystals, to distinguish them from supermirrors.) In particular, the refraction correction to Bragg's law, which has generally been neglected, may be sizeable; the component thicknesses of the bilayer must be derived on the basis of eqs. (3) and (4) for a correct response at low angles. Conversely, thicknesses derived from first-order low-angla diffraction data via the usual Bragg relation, $\lambda=2 d \theta$, rather than eqs. (3) and (4), will typically be underestimated by about $20 z$ for $d$ of order $20 \mathrm{~nm}$. This shift in the nominal Bragg angle offers a useful means of assessing in situ material densities, which are not known a priori in the multilayer, without having to measure absolute intensities; the positions alone of the mirror edge and of the first few orders of diffraction depend on scattering amplitude density, as well as on layer geometry.

In conclusion, the DTFM equations provide a straightforward prescription for designing supermirrors or bandpass filters of specified mean reflectivity, without any need for numerical trial and error, while optimizing the number of layers to minimize production costs.

\section{ACKNOWLEDGEMENTS}

This research was sponsored by the Division of Materials Sciences, U. S. Department of Energy, under contract DE-AC05-840R21400 with Martin Marietta Energy Systems, Inc.

\section{REFERENCES}

1. A.G. Klein and S.A. Werner, Rept. Prog. Phys. 46, 259-335 (1983).

2. M.L. Goldberger and F. Seitz, Phys. Rev. 71, 294-310 (1947).

3. R.W. James, The Optical Principles of the Diffraction of $X$-rays, Chapter 4, Bell, London (1954).

4. F. Mezei, Comm. Phys. 1, 81-85 (1976).

5. O.S. Heavens and H.M. Liddell, Appl. Optics. 5, 373-376 (1966).

6. F. Mezei and P.A. Dagleish, Comm. Phys. 2, 41-43 (1977).

7. A.G. Gukasov, V.A. Ruban, and M.N. Bedrizova, Sov. Tech. Phys. Lett. 3, 52-53 (1977).

8. S. Yamada, T. Ebisawa, N. Achiwa, T. Akiyoshi and S. Okamoto, Ann. Rep. Res. Reactor Inst. (Kyoto). 11, 8-27 (1978).

9. J. Schelten and K. Mika, Nucl. Instr. Meth. 160, 287-294 (1979).

10. O. Schărpf, A.I.P. Conf. Proc. 89, 182-189 (1982).

11. C.F. Majkrzak, Physica. 136B, 69-74 (1986).

12. V.F. Sears, Thermal Neutron Scattering Lengths and Cross-Sections for Condensed Matter Research, Report AECL-8490, Atomic Energy of Canada Ltd., Chalk River (1984).

13. B.E. Warren, X-Ray Diffraction, Addison-Wesley, London (1969).

14. O.S. Heavens, Optical Properties of Thin Solid Films, Butterworth, London (1955).

15. J. Penfold, Computer Simulation of Neutron Interference in Thin Films, Report 79PE36S, Institut Laue-Langevin, Grenoble (1979).

16. B.P. Schoenborn, D.L.D. Caspar and O.F. Kammerer, J. Appl. Cryst. 7, 508-510 (1974).

17. A.M. Saxena and B.P. Schoenborn, Acta Cryst. A33, 805-813 (1977). 